\title{
LEVEL SETS OF DENSITY CONTINUOUS FUNCTIONS
}

\author{
KRZYSZTOF CIESIELSKI AND LEE LARSON
}

\begin{abstract}
A function $f: \mathbf{R} \rightarrow \mathbf{R}$ is density continuous if it is continuous when both its range and domain are endowed with the density topology. The level sets of density continuous functions are characterised as those sets which are density closed and ambiguous.
\end{abstract}

\section{INTRODUCTION}

The density topology on the real line, $\mathbf{R}$, consists of all sets, $S$, such that each point of $S$ is a Lebesgue density point of $S$. The density topology is a completely regular refinement of the ordinary topology, which fails to be normal [4]. In this paper, we consider some properties of functions $f: \mathbf{R} \rightarrow \mathbf{R}$ which are continuous when the density topology is applied to both the range and domain. Such functions have been termed density continuous [6].

It has recently been shown that density continuous functions do not form a vector space, and there are monotone and even $C^{\infty}$ functions which are not density continuous [1]. On the other hand, all locally convex functions are density continuous [1] and density continuous functions are in the class Baire*1 [2].

In this paper, we answer a question posed by Ostaszewski [7] related to the properties of the class of density continuous functions, $\mathscr{C}_{D}$, when viewed as a semigroup. This question is:

Query. Given a density closed $\mathbf{G}_{\delta}$ subset $E$ of $\mathbf{R}$, is there an $f \in \mathscr{C}_{D}$ such that $E=f^{-1}(0)$ ?

It turns out that this question can be answered negatively. In $\S 2$, the level sets of density continuous functions are characterized as consisting of the sets which are closed in the density topology while being simultaneously $\mathbf{F}_{\sigma}$ and $\mathbf{G}_{\delta}$. This shows that any density closed set $E$ which is $\mathbf{G}_{\delta}$ but not $\mathbf{F}_{\sigma}$ is a counterexample to Ostaszewski's query.

We use the following notation:

$\mathbf{R}$-the set of real numbers;

$\mathbf{N}$-the set of natural numbers;

$|A|$-the Lebesgue measure of a measurable set $A \subset \mathbf{R}$;

$A^{c}$ - the complement of the set $A$;

Received by the editors December 15, 1989 and, in revised form, June 15, 1990.

1991 Mathematics Subject Classification. Primary 26A21. 
$\operatorname{int}(A)$-the interior of the set $A$;

$\operatorname{dist}(P, Q)=\inf \{|p-q|: p \in P$ and $q \in Q\}$-the distance between sets $P$ and $Q$;

$B(F, \varepsilon)=\{x: \operatorname{dist}(F, x)<\varepsilon\}$

$\bar{d}(A, x), \underline{d}(A, x), d^{+}(A, x), d^{-}(A, x)$, and $d(A, x)$-the upper, lower, right, left, and ordinary (respectively) densities of a set $A \subset \mathbf{R}$ at a point $x \in \mathbf{R}$;

$L_{f}(\alpha)=\{x \in \mathbf{R}: f(x)=\alpha\}$;

$\mathscr{C}_{D}$ - the set of all density continuous functions on $\mathbf{R}$.

Given $P \subset Q \subset \mathbf{R}$, we say $P$ is a portion of $Q$ if there exists an open interval $I$ such that $P=I \cap Q \neq \varnothing$.

\section{LEVEL SETS OF DENSITY CONTINUOUS FUNCTIONS}

The purpose of this section is to prove the characterization of the level sets of functions in $\mathscr{C}_{D}$. This is presented in Theorem 4. First, some preliminary results and definitions must be presented. Several of these results are interesting in their own right.

O'Malley [5] defined the class Baire* 1 to consist of all functions $f: \mathbf{R} \rightarrow \mathbf{R}$ such that for every perfect set $P$, there exists a portion $Q$ of $P$ such that $\left.f\right|_{Q}$ is continuous. The following theorem is known [2].

Theorem 1. $\mathscr{C}_{D} \subset$ Baire* $^{*}$.

Theorem 2. $\mathscr{C}_{D}$ is a lattice.

Proof. Let $f, g \in \mathscr{C}_{D}, h=\max \{f, g\}$, and $x_{0} \in \mathbf{R}$. Assume first that $h\left(x_{0}\right)=f\left(x_{0}\right)>g\left(x_{0}\right)$ and that $m \in\left(g\left(x_{0}\right), f\left(x_{0}\right)\right)$. If $G$ is a density neighborhood of $f\left(x_{0}\right)$ contained in $(m, \infty)$, then $H=f^{-1}(G) \cap g^{-1}((-\infty, m))$ is a density neighborhood of $x_{0}$ with the property that $h|H=f| H$. This implies that $h$ is density continuous at $x_{0}$. A symmetrical argument handles the case when $h\left(x_{0}\right)=g\left(x_{0}\right)>f\left(x_{0}\right)$.

Now, assume $f\left(x_{0}\right)=g\left(x_{0}\right)=h\left(x_{0}\right)$ and $G$ is a density neighborhood of $h\left(x_{0}\right)$. Both $f^{-1}(G)$ and $g^{-1}(G)$ are density neighborhoods of $x_{0}$, so $H=f^{-1}(G) \cap g^{-1}(G)$ is also a density neighborhood of $x_{0}$. If $x \in H$, then $f(x) \in G$ and $g(x) \in G$, so $h(x)=\max (f(x), g(x)) \in G$. From this, it follows that $h^{-1}(G) \supset H$ and $h$ is density continuous at $x_{0}$.

Therefore $\mathscr{C}_{D}$ is closed under the operation of taking the maximum of two functions. Since $\min (f(x), g(x))=-\max (-f(x),-g(x))$, we see $\mathscr{C}_{D}$ is closed under the minimization operation also. These two statements prove that $\mathscr{C}_{D}$ is a lattice.

The following lemma was proved by the present authors [3, Corollary 1].

Lemma 1. If $F$ is a closed set, then $f(x)=\operatorname{dist}(x, F) \in \mathscr{C}_{D}$.

The next theorem can be proved from a known result characterising the associated sets of Baire*1 functions due to $\mathrm{Pu}$ and $\mathrm{Pu}$ [8]. Since it is in the spirit of what follows, we include the following shorter proof.

Theorem 3. $A$ set $A$ is a level set for a function $f \in$ Baire $^{*} 1$ if and only if $A \in \mathbf{F}_{\sigma} \cap \mathbf{G}_{\delta}$. 
Proof. Suppose that $f \in$ Baire*1 and $\alpha \in \mathbf{R}$. Since Baire*1 is contained in Baire 1, it follows that $L_{f}(\alpha) \in \mathbf{G}_{\delta}$. To prove that $L_{f}(\alpha)$ is also in $\mathbf{F}_{\sigma}$, we use induction on $\zeta<\omega_{1}$ to define sequences $K_{\zeta}$ and $F_{\zeta}$ such that for every $\zeta<\omega_{1}$

(a) $K_{\zeta}=\overline{L_{f}(\alpha) \backslash \bigcup_{\eta<\zeta} F_{\eta}}$,

(b) $F_{\zeta} \subset L_{f}(\alpha) \cap K_{\zeta}$,

(c) $F_{\zeta}$ is relatively open in $K_{\zeta}$, and

(d) if $K_{\zeta} \neq \varnothing$, then $K_{\zeta+1}$ is a proper subset of $K_{\zeta}$.

By (a), we have defined $K_{\zeta}$, provided the $F_{\eta}$ are defined for all $\eta<\zeta$. In particular, $K_{0}=\overline{L_{f}(\alpha)}$.

Thus, let us assume that $K_{\zeta}$ is defined. If $K_{\zeta}=\varnothing$, then define $F_{\zeta}=\varnothing$. If $K_{\zeta} \neq \varnothing$, we can find, using the fact that $f \in$ Baire*1, a portion $F_{\zeta} \subset K_{\zeta}$ such that $f \mid F_{\zeta}$ is continuous. But, by (a), $L_{f}(\alpha) \cap F_{\zeta}$ is dense in $F_{\zeta}$, so $f \mid F_{\zeta} \equiv \alpha$ and, consequently, $F_{\zeta} \subset L_{f}(\alpha)$. This implies (b) and (c), and, together with (a), also (d). The construction is finished.

As the sequence $\left\{K_{\zeta}: \zeta<\omega_{1}\right\}$ of closed sets cannot be strictly decreasing, (d) implies that there exists an $\eta<\omega_{1}$ such that $K_{\eta}=\varnothing$; i.e., such that

$$
L_{f}(\alpha)=\bigcup_{\zeta<\eta} F_{\zeta} .
$$

But, (c) implies that each $F_{\zeta} \in \mathbf{F}_{\sigma}$, which immediately yields $L_{f}(\alpha) \in \mathbf{F}_{\sigma}$.

Next, suppose that $A \in \mathbf{F}_{\sigma} \cap \mathbf{G}_{\delta}, f(x)=\chi_{A}(x)$, and $P$ is a perfect set. By supposition, both $A$ and $A^{c}$ are $\mathbf{G}_{\delta}$ sets, so the Baire category theorem shows they both cannot be dense in $P$. Therefore, there must exist a portion $Q \subset P$ such that either $Q \subset A$ or $Q \subset A^{c}$. In either case, $f \mid Q$ is constant and therefore continuous. This shows that $f \in$ Baire*1 with $A=L_{f}(1)$.

Lemma 2. Let $A$ be a density closed set from $\mathbf{F}_{\sigma} \cap \mathbf{G}_{\delta}$ such that $A$ does not contain any infinite interval. Then there is a bounded open set $G$ in $\mathbf{R}$ such that $A \cap G$ is a nonempty compact set.

Proof. First, suppose that $A$ contains no open intervals. Assume the lemma is not true in this case. We claim that with this supposition, both $A$ and $A^{c}$ are dense $\mathbf{G}_{\delta}$ subsets of $\bar{A}$. This claim is clear for $A$. To prove it for $A^{c}$, let $I$ be any open interval such that $I \cap A \neq \varnothing$. Since $A$ contains no open intervals, there must exist a closed interval $[c, d] \subset I$ such that $[c, d] \cap A=(c, d) \cap A$ is nonempty and not closed. It follows easily from this that

$$
I \cap \bar{A} \backslash A \supset[c, d] \cap \bar{A} \backslash A \neq \varnothing,
$$

which implies the claim. But, this obviously contradicts the Baire category theorem. Therefore, the lemma must be true in this case.

Now, returning to the general case for $A$, we introduce an equivalence relation, $\sim$, on $A$ by $a \sim b \Leftrightarrow[a, b] \subset A$; i.e., all the closed and connected components of $A$ are the equivalence classes of $A / \sim$. There is obviously a set $B \subset \mathbf{R}$ which is homeomorphic to $A / \sim$; i.e., there is a nondecreasing function $h: A \rightarrow B$ such that $h(a)=h(b) \Leftrightarrow a \sim b . \quad B$ does not contain any interval, $B \in \mathbf{F}_{\sigma} \cap \mathbf{G}_{\delta}$, and $B$ is density closed. If $B \cap(a, b)$ is compact, then $h^{-1}((a, b))$ satisfies the conclusion of the lemma. 
The following lemma is an easy consequence of the preceding lemma.

Lemma 3. If $A$ is a nonempty density closed set from $\mathbf{F}_{\sigma} \cap \mathbf{G}_{\delta}$ such that $A$ does not contain any infinite interval, then there is a maximal family $\left\{U_{n}: n \in \mathbf{N}\right\}$ of pairwise disjoint bounded open sets such that $A \cap U_{n}$ is compact and nonempty.

Lemma 4. Let $A$ be a density closed set from $\mathbf{F}_{\sigma} \cap \mathbf{G}_{\delta}$ that does not contain any infinite interval. There is a countable decomposition $\left\{F_{\gamma}\right\}_{\gamma \in \Gamma}$ of $A$ into compact sets and a family $\left\{\varepsilon_{\gamma}\right\}_{\gamma \in \Gamma} \subset(0, \infty)$ such that

$$
B_{x}=\left\{\gamma \in \Gamma: x \in B\left(F_{\gamma}, \varepsilon_{\gamma}\right)\right\}
$$

is finite for all $x \in \mathbf{R}$ and

$$
\left\{B\left(F_{\gamma}, \varepsilon_{\gamma}\right) \backslash F_{\gamma}: \gamma \in \Gamma\right\}
$$

is locally finite in the density topology.

Proof. We will use induction to define sets $K_{\zeta}, F(\zeta, \eta)$, and $U(\zeta, \eta)$ for $\eta \in \mathbf{N}$ and $\zeta<\omega_{1}$ satisfying

(a) $F(\zeta, \eta)=K_{\zeta} \cap U(\zeta, \eta)$,

(b) $F(\zeta, \eta)$ is compact,

(c) $U(\zeta, \eta)$ are open and bounded,

(d) $K_{\zeta}=A \backslash \bigcup_{\alpha<\zeta} \bigcup_{\eta} U(\alpha, \eta)$,

(e) for fixed $\zeta$, the collection $\{U(\zeta, \eta): \eta \in \mathbf{N}\}$ is pairwise disjoint, and

(f) if $K_{\zeta} \neq \varnothing$, then there is an $\eta \in \mathbf{N}$ such that $K_{\zeta} \cap U(\zeta, \eta) \neq \varnothing$.

It follows from (d) that $K_{0}=A$. Properties (c) and (d) imply that every $K_{\zeta}$ satisfies the conditions of Lemma 3. Inductive application of Lemma 3 easily establishes (a)-(f). Moreover, $K_{\zeta}$ is a decreasing sequence of sets which are relatively closed in $A$, so there is an $\alpha<\omega_{1}$ such that for all $\beta>\alpha, K_{\beta}=K_{\alpha}$. Conditions (c), (d), and (f) guarantee that $K_{\alpha}=\varnothing$. Thus, putting

$$
\Gamma=\{(\zeta, \eta): \zeta<\alpha, \eta \in \mathbf{N}\},
$$

we can define $F_{\gamma}=F(\zeta, \eta)$ and $U_{\gamma}=U(\zeta, \eta)$ for all $\gamma=(\zeta, \eta) \in \Gamma$.

Decreasing $U_{\gamma}$ does not change the above properties as long as $F_{\gamma} \subset U_{\gamma}$ and $U_{\gamma}$ is open. Combining this with the fact that $F_{\gamma}$ is compact, we may assume that for every $\gamma$ there is a $\delta_{\gamma}>0$ such that $U_{\gamma}=B\left(F_{\gamma}, \delta_{\gamma}\right)$ and $\sum_{\gamma} \delta_{\gamma}<1$. Let $a_{\gamma}>0$ be such that $\sum_{\gamma} a_{\gamma}<1$ and choose $\varepsilon_{\gamma} \in\left(0, \delta_{\gamma}\right)$ such that

$$
\frac{\left|B\left(F_{\gamma}, \varepsilon_{\gamma}\right) \backslash F_{\gamma}\right|}{\delta_{\gamma}}<a_{\gamma} .
$$

First notice that for every $x \in \mathbf{R}$, the set $U_{x}=\left\{\gamma: x \in U_{\gamma}\right\}$ is finite. Otherwise, there is an infinite sequence $\gamma_{k}=\left(\zeta_{k}, \eta_{k}\right)$ such that $x \in U_{\gamma_{k}}$. It follows from (e) that $\zeta_{n} \neq \zeta_{m}$ whenever $n \neq m$. By passing to a subsequence, if necessary, we may assume that $\zeta_{k}$ is an increasing sequence.

Fix

$$
\delta=\operatorname{dist}\left(K_{\zeta_{1}}, x\right) \geq \operatorname{dist}\left(K_{\zeta_{0}+1}, x\right)>0,
$$

where the first inequality follows from the fact that $K_{\zeta}$ is a decreasing sequence and the second from (d). Since $\lim _{k} \delta_{\gamma_{k}}=0$, there is an $n>1$ such that 
$0<\delta_{\gamma_{m}}<\delta$ for all $m \geq n$. In particular,

$$
\delta_{\gamma_{n}}<\delta=\operatorname{dist}\left(K_{\zeta_{1}}, x\right) \leq \operatorname{dist}\left(F_{\gamma_{n}}, x\right) ;
$$

i.e., $x \notin B\left(F_{\gamma_{n}}, \delta_{\gamma_{n}}\right)=U_{\gamma_{n}}$. This contradiction establishes that $U_{x}$ is finite. This implies that $B_{x}$ is also finite, which establishes the first part of the lemma.

Let

$$
\Gamma_{1}=\Gamma \backslash B_{x}, \quad S=\bigcap_{\gamma \in B_{x}} B\left(F_{\gamma}, \varepsilon_{\gamma}\right) \backslash F_{\gamma}, \quad T=\bigcup_{\gamma \in \Gamma_{1}} B\left(F_{\gamma}, \varepsilon_{\gamma}\right) \backslash F_{\gamma},
$$

and $U=S \backslash T$. Evidently, $x \in U, S$ is open, and $U$ intersects only finitely many of the $B\left(F_{\gamma}, \varepsilon_{\gamma}\right) \backslash F_{\gamma}$. To finish the proof, it is enough to show that $U$ has full density at $x$. This is done by establishing that $d(T, x)=0$.

To do this, let $\varepsilon>0$. Choose $\Gamma_{2} \subset \Gamma_{1}$ such that $\Gamma_{1} \backslash \Gamma_{2}$ is finite and $\sum_{\gamma \in \Gamma_{2}} a_{\gamma}<\varepsilon$. Since $\varepsilon_{\gamma}<\delta_{\gamma}, k_{0}$ can be chosen such that for $k \geq k_{0}$,

$$
(x, x+1 / k) \cap\left(B\left(F_{\gamma}, \varepsilon_{\gamma}\right) \backslash F_{\gamma}\right)=\varnothing \quad \forall \gamma \in \Gamma_{1} \backslash \Gamma_{2} .
$$

Thus,

$$
\begin{aligned}
& \left|(x, x+1 / k) \cap \bigcup_{\gamma \in \Gamma_{1}}\left(B\left(F_{\gamma}, \varepsilon_{\gamma}\right) \backslash F_{\gamma}\right)\right| /(1 / k) \\
& \quad \leq k \sum_{\gamma \in \Gamma_{2}}\left|(x, x+1 / k) \cap\left(B\left(F_{\gamma}, \varepsilon_{\gamma}\right) \backslash F_{\gamma}\right)\right| \leq \sum_{\gamma \in \Gamma_{2}} a_{\gamma} / k<\varepsilon .
\end{aligned}
$$

The first inequality in (2) is obvious. The second inequality in (2) is handled in two cases. If $\delta_{\gamma} \leq 1 / k$, then by (1)

$$
\left|(x, x+1 / k) \cap\left(B\left(F_{\gamma}, \varepsilon_{\gamma}\right) \backslash F_{\gamma}\right)\right| \leq\left|B\left(F_{\gamma}, \varepsilon_{\gamma}\right) \backslash F_{\gamma}\right| \leq a_{\gamma} \delta_{\gamma} \leq a_{\gamma} / k .
$$

If $\delta_{\gamma}>1 / k$, then

$$
1 / k<\delta_{\gamma}=\operatorname{dist}\left(F_{\gamma}, U_{\gamma}^{c}\right) \leq \operatorname{dist}\left(F_{\gamma}, x\right)
$$

by the definitions of $F_{\gamma}$ and $U_{\gamma}$ with $x \notin U_{\gamma}$. Hence,

$$
\begin{aligned}
\left|(x, x+1 / k) \cap\left(B\left(F_{\gamma}, \varepsilon_{\gamma}\right) \backslash F_{\gamma}\right)\right|= & \left|(x, x+1 / k) \cap\left(B\left(F_{\gamma} \cap(-\infty, x], \varepsilon_{\gamma}\right) \backslash F_{\gamma}\right)\right| \\
& +\left|(x, x+1 / k) \cap B\left(F_{\gamma} \cap[x, \infty), \varepsilon_{\gamma}\right) \backslash F_{\gamma}\right| .
\end{aligned}
$$

The first term on the right-hand side is the empty set because $\varepsilon_{\gamma}<\delta_{\gamma}$ $<\operatorname{dist}\left(x, F_{\gamma}\right)$ and from the assumption that $1 / k<\delta_{\gamma}$. Letting $y=$ $\inf \left(F_{\gamma} \cap[x, \infty)\right)$, we see that

$$
\left|(x, x+1 / k) \cap\left(B\left(F_{\gamma}, \varepsilon_{\gamma}\right) \backslash F_{\gamma}\right)\right|=\left|(x, x+1 / k) \cap\left(y-\varepsilon_{\gamma}, y\right)\right| .
$$

In case $y=\infty$, the right-hand side vanishes and the conclusion is obvious. Assume $y \in \mathbf{R}$. Notice that $y>x+1 / k, y-\delta_{\gamma}>x$, and $y-\delta_{k}+1 / k<y$ because

$$
\operatorname{dist}(x, y) \geq \operatorname{dist}\left(x, F_{\gamma}\right) \geq \delta_{\gamma}>1 / k
$$


Hence,

$$
\begin{aligned}
\left|\left(x, x+\frac{1}{k}\right) \cap\left(y-\varepsilon_{\gamma}, y\right)\right| & \leq\left|\left(y-\delta_{\gamma}, y-\delta_{\gamma}+\frac{1}{k}\right) \cap\left(y-\varepsilon_{\gamma}, y\right)\right| \\
& =\frac{\left|\left(y-\delta_{\gamma}, y-\delta_{\gamma}+1 / k\right) \cap\left(y-\varepsilon_{\gamma}, y\right)\right|}{k\left|\left(y-\delta_{\gamma}, y-\delta_{\gamma}+1 / k\right)\right|} \\
& \leq \frac{\left|\left(y-\delta_{\gamma}, y\right) \cap\left(y-\varepsilon_{\gamma}, y\right)\right|}{k\left|\left(y-\delta_{\gamma}, y\right)\right|} \\
& =\frac{\varepsilon_{\gamma}}{k \delta_{\gamma}} \leq \frac{\left|B\left(F_{\gamma}, \varepsilon_{\gamma}\right) \backslash F_{\gamma}\right|}{k \delta_{\gamma}} \leq \frac{a_{\gamma}}{k} .
\end{aligned}
$$

The second inequality in the expression written above is justified by noting that if $0<a<b$ and $c>0$, then $a / b<(a+c) /(b+c)$. Therefore $d^{+}(T, x)=0$.

A similar argument can be presented for the left density. The lemma follows from these densities.

Theorem 4. The level sets of density continuous functions are precisely the density closed sets which are in $\mathbf{F}_{\sigma} \cap \mathbf{G}_{\delta}$.

Proof. In light of Theorems 3 and 1 we see that the level sets of any density continuous function are in $\mathbf{F}_{\sigma} \cap \mathbf{G}_{\delta}$ and are density closed. To finish the proof of the theorem, it suffices to show that given a density closed set $A \in \mathbf{F}_{\sigma} \cap \mathbf{G}_{\delta}$, there is a function $f \in \mathscr{C}_{D}$ such that $A=L_{f}(0)$.

First assume that $A$ does not contain any infinite interval. Let $\Gamma, B_{x}, F_{\gamma}$, and $\varepsilon_{\gamma}$ be as in Lemma 4. Define

$$
h_{\gamma}(x)=\operatorname{dist}\left(x, F_{\gamma}\right) / \varepsilon_{\gamma},
$$

so that $h_{\gamma} \equiv 0$ on $F_{\gamma}$ and $h_{\gamma} \geq 1$ on $B\left(F_{\gamma}, \varepsilon_{\gamma}\right)^{c}$. According to Lemma $4, B_{x}$ is finite for all $x$, so that a function $f$ may be defined as

$$
f(x)=\min _{\gamma \in \Gamma}\left\{1, h_{\gamma}(x)\right\} .
$$

Evidently, in light of Lemma $1, h_{\gamma}$ and 1 are density continuous. Also, $f^{-1}(0)$ $=\bigcup_{\gamma \in \Gamma} F_{\gamma}=A$. It suffices to prove that $f$ is density continuous.

Let $x \in \mathbf{R}$. According to Lemma 4 there is a density open set $G$ containing $x$ such that

$$
G_{x}=\left\{\gamma: G \cap B\left(F_{\gamma}, \delta_{\gamma}\right) \backslash F_{\gamma} \neq \varnothing\right\}
$$

is finite. We consider two cases.

Case 1. Assume $x \notin A$. Then Theorem 2 shows that

$$
f \mid G=\min _{\alpha \in G_{x}}\left\{1, h_{\alpha}\right\}
$$

is density continuous on $G$.

Case 2. Let $x \in A$. If $g=\min _{\gamma \in G_{x}}\left\{1, h_{\gamma}\right\}$, then, as in Case $1, g$ is density continuous. Suppose $D$ is a density neighborhood of $f(x)=0$. Then

$$
\begin{aligned}
f^{-1}(D) & =\left[f^{-1}(D) \cap A^{c}\right] \cup\left[f^{-1}(D) \cap A\right] \supset\left[f^{-1}(D) \cap G \cap A^{c}\right] \cup A \\
& =\left[g^{-1}(D) \cap G \cap A^{c}\right] \cup A \supset g^{-1}(D) \cap G .
\end{aligned}
$$

This case is now clear because $g^{-1}(D) \cap G$ is a density neighborhood of $x$. 
Finally, if $A$ does contain an infinite interval, let $-\infty \leq a<b \leq \infty$ be such that $[a, b] \cap A$ does not contain an infinite interval and such that $\mathbf{R} \backslash(a, b) \subset$ $A$. Then, if $f$ is defined for $A \cap[a, b]$ as above, the function

$$
f_{0}(x)=\min \{f(x), \operatorname{dist}(x, \mathbf{R} \backslash(a, b))\}
$$

satisfies the conclusion of the theorem.

Corollary 1. The associated sets of density continuous functions are precisely the density open sets which are in $\mathbf{F}_{\sigma} \cap \mathbf{G}_{\delta}$.

Proof. If $f$ is any real function and $\alpha \in \mathbf{R}$, define $g=\max \{f, \alpha\}$. Since $\{x: f(x)>\alpha\}=\left(L_{g}(\alpha)\right)^{c}$, this corollary follows from Theorems 2 and 4.

\section{REFERENCES}

1. Krzysztof Ciesielski and Lee Larson, The space of density continuous functions, Acta Math. Hungar. 58 (1991).

2. Krzysztof Ciesielski, Lee Larson, and Krzysztof Ostaszewski, Density continuity versus continuity, Forum Math. 1 (1989), 1-11.

3. _ Differentiability and density continuity, Real Anal. Exchange 15 (1989-90), 239-247.

4. C. Goffman, C. J. Neugebauer, and T. Nishiura, The density topology and approximate continuity, Duke Math. J. 28 (1961), 497-506.

5. R. J. O'Malley, Baire*1 Darboux functions, Proc. Amer. Math. Soc. 60 (1976), 187-192.

6. K. Ostaszewski, Continuity in the density topology, Real Anal. Exchange 7 (1982), 259-270.

7. __ Semigroups of density continuous functions, Real Anal. Exchange 14 (1988-89), 104114.

8. H. W. Pu and H. H. Pu, Associated sets of Baire*1 functions, Real Anal. Exchange 8 (1982$83), 479-485$. 26506

Department of Mathematics, West Virginia University, Morgantown, West Virginia

Department of Mathematics, University of Louisville, Louisville, Kentucky 40292 